\title{
Effect of imaging and catheter characteristics on clinical outcome for patients in the PRECISE study
}

\author{
Sabine Mueller $\cdot$ Mei-Yin Polley $\cdot$ Benjamin Lee $\cdot$ Sandeep Kunwar $\cdot$ \\ Christoph Pedain · Eva Wembacher-Schröder · Stephan Mittermeyer • \\ Manfred Westphal · John H. Sampson • Michael A. Vogelbaum • \\ David Croteau $\cdot$ Susan M. Chang
}

Received: 14 January 2010/Accepted: 24 May 2010/Published online: 20 June 2010

(C) The Author(s) 2010. This article is published with open access at Springerlink.com

\begin{abstract}
The PRECISE study used convection enhanced delivery (CED) to infuse IL13-PE38QQR in patients with recurrent glioblastoma multiforme (GBM) and compared survival to Gliadel Wafers (GW). The objectives of this retrospective evaluation were to assess: (1) catheter positioning in relation to imaging features and (2) to examine the potential impact of catheter positioning, overall catheter placement and imaging features on long term clinical outcome in the PRECISE study. Catheter positioning and overall catheter placement were scored and used as a surrogate of adequate placement. Imaging studies obtained on day 43 and day 71 after resection were each retrospectively reviewed. Catheter positioning scores, catheter overall placement scores, local tumor control and imaging change scores were reviewed and correlated using Generalized Linear Mixed Models. Cox PH regression analysis was
\end{abstract}

PRECISE Study Group sites and Principal Investigators are noted in the Appendix section.

This study was conducted for the PRECISE STUDY GROUP.

S. Mueller ( $\square)$

Department of Neurology, University of San Francisco,

350 Parnassus Ave, Suite 609, San Francisco, CA 94117, USA

e-mail: muellers@neuropeds.ucsf.edu

M.-Y. Polley $\cdot$ S. Kunwar · S. M. Chang

Department of Neurosurgery, University of San Francisco,

400 Parnassus Ave, San Francisco, CA 94117, USA

B. Lee

Department of Neuroradiology, University of San Francisco,

555 Parnassus Ave, San Francisco, CA 94117, USA

C. Pedain - E. Wembacher-Schröder - S. Mittermeyer BrainLAB AG, Kapellenstrasse 12, 85622 Feldkirchen, Germany used to examine whether these imaging based variables predicted overall survival (OS) and progression free survival (PFS) after adjusting for age and KPS. Of 180 patients in the CED group, 20 patients did not undergo gross total resection. Of the remaining 160 patients only $53 \%$ of patients had fully conforming catheters in respect to overall placement and $51 \%$ had adequate catheter positioning scores. Better catheter positioning scores were not correlated with local tumor control $(P=0.61)$ or imaging change score $(P=0.86)$. OS and PFS were not correlated with catheter positioning score (OS: $P=0.53$; PFS: $P=0.72$ respectively), overall placement score (OS: $P=0.55$; PFS: $P=0.35$ ) or imaging changes on day 43 MRI $(P=0.88)$. Catheter positioning scores and overall catheter placement scores were not associated with clinical outcome in this large prospective trial.

Keywords Convection enhanced delivery . PRECISE study · Imaging · Catheter scores · Recurrent glioblastoma

M. Westphal

Klinik und Poliklinik fuer Neurochirugie, Martinistrasse 52, 20246 Hamburg, Germany

J. H. Sampson

Division of Neurosurgery, Department of Surgery, Duke

University, Durham, NC 27710, USA

M. A. Vogelbaum

Brain Tumor and Neuro-Oncology Center, Cleveland Clinic, 9500 Euclid Avenue, Cleveland, OH 44195, USA

D. Croteau

NeoPharm Inc, 101 Waukegan Rd, Suite 970, Lake Bluff,

IL 60044, USA 


\section{Introduction}

Recurrent glioblastoma multiforme (GBM) has a dismal prognosis with a median survival of 6 months despite multi-modality treatment strategies. The introduction of temozolamide led to prolonged survival of newly diagnosed patients with GBM after radiation and surgical resection to 15 months [1] but therapeutic options for recurrent GBM are limited. Gliadel wafers $(\mathrm{GW})$ are currently the only Food and Drug Administration (FDA) approved local treatment for recurrent GBM. The restrictive blood brain barrier (BBB) remains a tremendous challenge for the development of systemically delivered therapeutics. Ongoing studies are currently investigating the role of regional delivery systems for these patients including convection enhanced delivery (CED) combined with targeted therapy such as cintredekin-besudotox (CB) and TP-38, a recombinant protein targeting the epidermal growth factor receptor [2, 3]. CB, also referred to as IL13PE38QQR, has been tested in prior phase I studies using CED as delivery methodology. $\mathrm{CB}$, a recombinant cytotoxin composed of the human interleukin -13 (IL-13) and a truncated form of the Pseudomonas aeruginosa Exotoxin A, has been shown to be highly toxic to cells expressing the IL-13 receptor $[4,5]$. Pseudomonas Exotoxin A kills mammalian cells by catalyzing irreversible ADP-ribosylation and inactivation of the elongation factor 2 necessary for protein synthesis. Human GBMs express high levels of the IL-13 receptor in comparison to normal brain and therefore this genetically engineered drug should be highly specific to tumor cells $[6,7]$. Investigations to assess if CED can successfully distribute IL13-PE38QQR demonstrated that co-infusion of IL13-PE38QQR with ${ }^{123} \mathrm{I}-$ labeled human serum albumin (HAS) in patients with GBM led under ideal circumstances to adequate tissue distribution of the drug [8]. The authors also identified that proximity to subarachnoid space and placement close to an ependymal surface has a significant detrimental influence on the drug delivery. Specific catheter positioning placement guidelines were therefore proposed based on these findings with an emphasis on adequate depth from brain surface, sulcus or resection cavity as well as distance from ependymal and pial surfaces. Timing of catheter placement is also important for the accuracy of the positioning. Edema, fluid shifts and re-expansion after surgical resection of the tumor limits the use of pre-surgical MRIs for catheter placement planning. Therefore most neurosurgeons using CED as delivery method will plan a second procedure for catheter implantation based on post resection images. Prior phase I studies found that accurate catheter positioning based on these guidelines seem to be a critical factor to improve OS assuming that accurately positioned catheters result in better drug distribution.
These studies also proved that CB could be infused safely via CED in patients after tumor resection [9]. The imaging changes seen on MRIs of patients treated with $\mathrm{CB}$ via CED are summarized by Parney et al. [10] and a specific scoring system has been established. It is thought that higher grades of imaging changes are due to a necrotic and inflammatory processes involving also normal brain. The underlying mechanism is not well understood but higher concentration of the drug could potentially lead to nontargeted uptake of $\mathrm{CB}$ and therefore also harm normal tissue. However, others have shown that the development of new T2/FLAIR signal abnormalities after delivery of $\mathrm{CB}$ via $\mathrm{CED}$ is an indication of successful drug delivery using concurrent infusion of $\mathrm{CB}$ and ${ }^{123}$ I-labeled HSA in combination with SPECT analysis [11]. The encouraging results from prior phase I studies led to the design of the multicenter phase III trial. The PRECISE study is the largest surgically based randomized clinical trial to date using CED in comparison to other local therapy in recurrent GBM. No survival advantage in patients treated with IL13-PE38QQR compared to GW was identified with a median survival of 36.4 weeks for $\mathrm{CB}$ and 35.3 weeks for $\mathrm{GW}(P=0.476)$ with hazard ratio of $0.89(95 \% \mathrm{CI}$ : $0.67-1.18$ ). Although progression free survival (PFS) was not an endpoint centrally reviewed in this trial, the study reported an improved PFS of 17.7 weeks for CB over a PFS of 11.4 weeks for GW $(P=0.008)$. To further elucidate which factors are essential to respond to this type of therapy, we assessed MRIs of all patients receiving IL13PE38QQR in respect to catheter positioning scores, overall catheter placement scores, local recurrence of disease, imaging changes associated with the therapy as well as the effect of these variables on clinical outcome.

\section{Materials and methods}

\section{Cintredekin besudotox (CB)}

The sequence encoding IL13-PE38QQR was created by Dr. Debinski and further developed by Dr. Raj K. Puri, M.D., Ph.D. in the department of Tumor Vaccines and Biotechnology Branch, Center for Biologics Evaluation and Research, FDA. The sequence was incorporated into a plasmid at Advanced BioScience Laboratories (Kensington, MD) and later at Diosynth (Morrisville, MC). Escherichia coli bacteria were transformed with a plasmid containing the $\mathrm{CB}$ sequence, protein synthesis was induced and expanded. $\mathrm{CB}$ was purified as described prior under current good manufacturing practices [6]. CB was infused at a concentration of $0.5 \mu \mathrm{g} / \mathrm{ml}$ at a total rate of $0.750 \mathrm{ml} / \mathrm{h}$ for $96 \mathrm{~h}$ after catheter placement was confirmed. 
Clinical trial design

The PRECISE study is an international, multicenter study across the U.S, Canada, Europe and Israel. Adult patients with recurrent supratentorial GBM, histologically confirmed at initial presentation, were eligible for the trial. The patients had to be surgical candidates with an intent of gross total resection (GTR), defined as more than $95 \%$ of the total volume of the enhancing lesion. Other inclusion criteria consisted of Karnofsky performance score $\geq 70$ as well as prior radiation therapy completed $\geq 4$ weeks prior to study entry. Patients who had multifocal disease, subependymal or leptomeningeal spread, evidence of tumor crossing the midline, or who showed signs of significant increased intracranial pressure, had uncontrolled seizures, or required emergent palliative treatment were excluded. Survival was defined as the number of days from the date of randomization to the date of death or last known alive date. PFS was defined as the number of days from the date of randomization to the date of tumor progression as determined by the investigator. For detail see Kunwar et al. [12].

\section{Imaging overview}

All patients underwent pre-resection contrast MRI imaging (with and without gadolinium) within 7 days prior to surgery. Within $72 \mathrm{~h}$ after resection all patients underwent an MRI scan (slices $<3 \mathrm{~mm}$ without spacing) to assess the extent of resection. These images were also used to plan for guidelines-compliant catheter trajectories. Based upon this planning MRI scan, on the day of catheter placement, two to four catheters were stereotactically placed by the treating neurosurgeon. To confirm proper catheter positioning, a non-contrast CT scan using slices $\leq 3 \mathrm{~mm}$ without spacing was performed prior to start the IL13-PE38QQR infusion. Follow-up contrast MR images were obtained at day 43 and day 71 post infusion and consistently collected for central review for all patients.

\section{Catheter planning and placement}

The surgical planning procedure for the infusion catheters was performed using the MRI scan obtained within $72 \mathrm{~h}$ of resection. The images were reviewed on the planning computer station or the surgical navigation station. Virtual trajectories were generated based upon the guidelines outlined in Table 1. Each trajectory was reviewed in Probe's Eye View in order to ensure adherence to the placement guidelines. Barium impregnated infusion catheters (Vygon, Norristown, PA, USA), not specifically designed for CED, were inserted in the operating room under local or general anesthesia. Using a sterile frameless or frame based stereotactic placement technique, two to four catheters were inserted into the brain parenchyma based on the surgical plan. For frameless insertion, the guideline was to use rigidly mounted holding devices rather than free-hand approaches in order to minimize deviations of actual versus planned catheter trajectories. The stylets from the catheters were slowly removed under constant irrigation so that the vacuum in the catheter would draw fluid down into the catheter and reduce the amount of air in the infusion system. Catheters were then tunneled beneath the scalp to reduce the risk of infection. Each catheter was connected to a separate micro infusion pump (MEDEX 3500 or equivalent). Placement was confirmed on CT imaging within $24 \mathrm{~h}$ of placement. The technique for placement was however not centrally mandated and was left to the discretion of each investigator. Infusion was started within $24 \mathrm{~h}$ after catheter placement. All members of the clinical team involved in the study received on-site and off-site training to gain basic proficiency and comfort level in using CED for the PRECISE Trial (BrainLAB AG, Feldkirchen, Germany). Investigators were not permitted to enroll a second patient until their first patient's plan and catheter placements were reviewed and deemed acceptable. In all cases, near real-time, but post-treatment, analysis of catheter planning and placement was performed and feedback was provided to the surgeon. Major deviations from the guidelines or from the catheter plan resulted in a requirement for retraining prior to enrollment of another patient.

A separate post-resection procedure for placement of catheters was chosen to improve the accuracy of catheter positioning by avoiding the effects of postoperative edema as well as brain shift and re-expansion on catheter positioning that can occur with placement at the time of resection.

\section{Central case review of catheter placement}

A central case review procedure to assess catheter placement was established in order to optimize clinical trial management and to improve interpretability of patient outcome. For this review BrainLAB collected anonymous data for each patient (post-resection/catheter planning MRI, post-catheter placement CT scan) in DICOM format at every site after treatment was completed. BrainLAB imported all available data to the iPlan ${ }^{\circledR}$ Flow planning and simulation software (BrainLAB AG, Feldkirchen, Germany). The planning functionality was used to add a virtual trajectory for every catheter depicted on the CT scan. To visualize these trajectories on all available images, CT and MRI data were overlaid using the image fusion functionality in the iPlan ${ }^{\circledR}$ Flow planning and simulation software, which is described in more detail elsewhere [8, 13, 14]. 
Three CED experienced neurosurgeons (MV, MW and SK) reviewed all images for each individual patient independently after the study was completed. Each catheter was measured and rated for compliance with the placement guidelines for catheter position geometry (catheter positioning score) and overall catheter placement for each patient (overall catheter placement score). Tables 1 and 2 summarize the catheter positioning scoring scheme based on study guidelines. The overall placement with respect of localization of the catheters to T2/FLAIR signal abnormality, resection cavity as well to other catheters was assessed in the central review process as well. Tables 3 and 4 summarize the overall catheter placement scoring system.

Table 1 Criteria for catheter positioning

\begin{tabular}{ll}
\hline Criterion & Definition \\
\hline A & $\begin{array}{r}\text { Depth } \geq 25 \mathrm{~mm} \text { from brain surface or any deep sulcus, or } \\
\text { from resection cavity wall if placed through the resection } \\
\text { cavity }\end{array}$ \\
& Catheter tip $\geq 5 \mathrm{~mm}$ from any pial surfaces \\
B & Catheter tip $\geq 10 \mathrm{~mm}$ from the resection cavity walls or \\
any ependymal surfaces
\end{tabular}

Table 2 Scoring system for assessment of catheter positioning

\begin{tabular}{ll}
\hline Score & Definition \\
\hline 0 & Poor: criterion A not fulfilled (regardless of other criteria) \\
1 & Fair: criteria A and either B or C fulfilled \\
2 & Good: all three criteria fulfilled \\
\hline
\end{tabular}

Table 3 Criteria for overall catheter placement

\begin{tabular}{ll}
\hline Criterion & Definition \\
\hline I & $\begin{array}{c}\text { Target includes areas of T2/FLAIR abnormalities and } \\
\text { largest adjacent area of white matter }\end{array}$ \\
II & $\begin{array}{c}\text { Catheter tips are } \geq 20 \mathrm{~mm} \text { apart from each other } \\
\text { III }\end{array}$ \\
& $\begin{array}{l}\text { If any residual, solid contrast-enhancing disease is present } \\
\text { the catheter tip is positioned adjacent but not into it }\end{array}$ \\
\hline
\end{tabular}

Table 4 Scoring system for overall catheter placement

\begin{tabular}{ll}
\hline Score & Definition \\
\hline $\mathrm{F}=$ fully conforming & $\begin{array}{l}\text { Criteria I, II and III (if applicable) fulfilled } \\
\mathrm{P}=\text { partially } \\
\text { conforming }\end{array}$ \\
$\begin{array}{l}\text { Only one criteria fulfilled or criteria I and II } \\
\text { fulfilled with criteria III failed } \\
\text { (if applicable) }\end{array}$ \\
$\mathrm{N}=$ non-conforming & No criteria fulfilled \\
\hline
\end{tabular}

Retrospective imaging analysis

Images obtained on day 43 and day 71 after resection during the PRECISE trial were each retrospectively reviewed at the University of California, San Francisco (UCSF) for patients treated with IL13-PE38QQR via CED with the objectives to: (1) assess catheter placement in relation to imaging features and (2) examine the potential impact of catheter positioning score, overall catheter placement score and imaging features on long term clinical outcome. A trained UCSF neuroradiologist (BL), who was blinded to the clinical outcome, confirmed the extent of tumor resection for each patient as well as progression or recurrence of disease on either day 43 or day 71 MRI. Imaging change scores were assessed for each catheter as outlined in Table 5 [10]. Local tumor control was defined as absence of tumor recurrence or progression within $1 \mathrm{~cm}$ radius of the trajectory length of the catheter. Local tumor control was assessed for each catheter. Progression was defined as existing tumor that was enlarging over interval MRI scans. Recurrence was defined as new nodular or suspicious enhancement at day 43 or 71 after GTR was confirmed on immediate postoperative MRI.

Statistical analyses

Patients with extent of resection less than $95 \%$ were excluded from the analysis to ensure that the statistical analyses were based on the intended population. To explore whether the catheter positioning score predicted local tumor control or imaging change scores on day 43 and day 71 MRIs, we employed the extended versions of logistic regression model and proportional odds model. These types of statistical models belong to the class of Generalized Linear Mixed Models (GLMM), which takes into proper consideration the correlation arising from multiple catheters (two to four) in each of the patients. Similarly, a

Table 5 Imaging change score

\begin{tabular}{|c|c|}
\hline $\begin{array}{l}\text { Imaging change } \\
\text { score }\end{array}$ & MRI changes ${ }^{\mathrm{a}}$ \\
\hline I & $\begin{array}{l}\text { Hyperintense signal abnormality on FLAIR relatec } \\
\text { to catheter tract or tip only. No new contrast- } \\
\text { enhancement }\end{array}$ \\
\hline II & $\begin{array}{l}\text { Mild contrast-enhancement }(<1.0 \mathrm{~cm} \text { or linear }) \\
\text { related to catheter tract or tip }\end{array}$ \\
\hline III & $\begin{array}{l}\text { Moderate contrast-enhancement }(1.0-3.0 \mathrm{~cm}) \\
\text { related to catheter tract or tip }\end{array}$ \\
\hline IV & $\begin{array}{l}\text { Extensive contrast-enhancement }(>3.0 \mathrm{~cm}) \text { related } \\
\text { to catheter tract or tip, with or without central } \\
\text { hypointensity }\end{array}$ \\
\hline
\end{tabular}

${ }^{a}$ Contrast-enhancing lesions diameter include the central hypointensity, if present 
generalized logistic regression model was used to examine if imaging change score was associated with local tumor control. Both catheter positioning score and imaging change score were treated as categorical variables when included as covariates in the model. These analyses were performed using STATA version 10.0 [15]. The Survival package in $\mathrm{R}$ version 2.7.1 was utilized to evaluate whether any imaging-based variable predicted long term survival [16]. For these analyses, two or more catheters optimally positioned (i.e. catheter positioning score 2; see Tables 1 and 2) were used as the categorical cut-off. For overall catheter placement score, patients assessed as "non-conforming" were collapsed with the "partially conforming" category due to limited number in the former category. Similarly, two or more catheters with imaging change score graded II or above (see Table 5) were used as the criterion for the purpose of dichotomization. For the local tumor control variable, patients were categorized into either achieving local tumor control in all catheters or having at least one local catheter failure. To assess whether catheter positioning score or overall catheter placement predicted long term clinical outcome, the Cox proportional hazards (Cox PH) model was used to allow for the adjustment of the known prognostic markers of age and KPS. Further, to examine the impact of day 43 and day 71 imaging variables (i.e. local tumor control and imaging change score) on clinical outcomes, we used a landmark analysis approach based on Cox PH model. For example, to evaluate whether local tumor control on day 43 MRIs predicted OS or PFS, all patients alive with known local tumor control status on day 43 were included in the analysis. Survival was counted as number of days from the date that day 43 MRI was performed until date of death due to any cause and Cox $\mathrm{PH}$ model was then used to adjust for factors including age and KPS. Adjustments for multiple comparisons was made by choosing $P$ values of $<0.01$ to indicate statistical significance.

\section{Results}

Distribution of catheter scores and imaging grading scores in the CB treated population

Among 183 patients in the CED ITT population, the extent of resection could not be evaluated in three patients. Twenty patients had significant residual disease and were excluded from this analysis. This differs from the analysis by Sampson et al. [17], who included all patients treated in the PRECISE trial with CB. The analysis was based on 160 patients. Of note, only 50 patients had no evidence of any residual disease defined as contrast enhancing lesion after surgery per USCF review. Seventy-five patients (47\%) treated with IL13-PE38QQR via CED had three catheters placed, 63 (39\%) had four catheters, and only 16 patients had two catheters. With respect to the overall catheter placement score, 83 patients (54\%) were judged to fulfill all of the criteria as outlined in Tables 3 and 4 (i.e. fully conforming), while 65 patients $(40 \%)$ only partially fulfilled the criteria and 5 patients $(3 \%)$ did not fulfill any.

Table 6 summarizes the distribution of each imaging based variable on the catheter level along with the patient number for which the assessment of each variable was available. The catheter scores were available for 154 patients with 509 catheters placed in total. Out of these catheters, $258(51 \%)$ were determined as fulfilling all catheter positioning criteria and were assigned an optimal score of 2, 59 catheters (12\%) were scored 1 and 192 catheters $(38 \%)$ scored 0 . At the patient level (data not shown), 84 out of 154 patients $(55 \%)$ had 2 or more catheters with an optimal score of 2. Local tumor control assessment for each catheter was available for 119 and 102 patients on day 43 and day 71, respectively. Local tumor control was achieved in $310 / 384$ catheters $(81 \%)$ on day 43 MRI and 267/329 catheters (81\%) on day 71 MRI achieved local tumor control per UCSF designation. Furthermore,

Table 6 Distribution of catheter positioning score, local tumor control and imaging change score

\begin{tabular}{lc}
\hline Variable & Number of catheters $(\%)$ \\
\hline Catheter positioning score $(\#$ pts $=154)$ & $192(38)$ \\
0 & $59(12)$ \\
1 & $258(51)$ \\
2 & \\
Local tumor control & \\
Day 43 (\# pts $=119)$ & $74(19)$ \\
No & $310(81)$ \\
Yes & \\
Day $71(\#$ pts $=102)$ & $62(19)$ \\
No & $267(81)$ \\
Yes & \\
Image change score & \\
Day $43(\#$ pts $=15)$ & $40(11)$ \\
0 & $92(26)$ \\
1 & $178(50)$ \\
2 & $37(10)$ \\
3 & $6(2)$ \\
4 & $38(13)$ \\
Day $71(\#$ pts = 99) & $73(25)$ \\
0 & \\
1 & $36(47)$ \\
2 & \\
3 & \\
4 & \\
\hline & \\
\hline
\end{tabular}


306 catheters were available for review of local tumor control on both day 43 and day 71, among which $94 \%$ had the same assessments on both days (one-sided $95 \%$ lower confidence limit $=91 \%$ ). At the patient level (data not shown), 82 patients (69\%) on day 43 and 69 patients (68\%) on day 71 achieved local tumor control on all catheters placed. Imaging change score due to $\mathrm{CB}$ via $\mathrm{CED}$ was graded according to guidelines outlined in Table 5 [10]. Imaging change scores on both day 43 and day 71 were evaluated for 271 catheters, among which $80 \%$ had the same imaging change scores on both days (one-sided $95 \%$ lower confidence limit $=76 \%$ ). The majority of catheters had low to moderate imaging grading scores: $87 \%$ and $85 \%$ scored II or below on day 43 and day 71, respectively. The distribution of imaging change score found in our study resembles that previously reported by Kunwar et al. [9] based on earlier phase I studies using $\mathrm{CB}$ at a similar concentration.

Correlative analysis of catheter scores, local tumor control and imaging changes

A prior study of patients treated with $\mathrm{CB}$ via $\mathrm{CED}$ suggested that the development of new T2/FLAIR signal abnormality is associated with drug distribution [11]. We therefore used the imaging change score as a surrogate for drug distribution in this imaging analysis. We hypothesized that better catheter scores may be correlated with better tumor control and higher imaging grading scores. Table 7 summarizes the correlative analysis of catheter positioning score, local tumor control and imaging change score based on day 43 and day 71 MRI data. We found that, contrary to our premise, catheter positioning score was neither predictive of local tumor control $(P=0.61$ and $P=0.66$ on day 43 and day 71 respectively) nor predictive of imaging change score $(P=0.86$ and $P=0.014$ on day 43 and day 71 respectively). In addition, imaging change score was not correlated with local tumor control either on day 43 $(P=0.2)$ or day $71(P=0.24)$.

Effect of catheter positioning score, overall catheter placement score and imaging change score on clinical outcome

The composite analysis of prior phase I trials by Kunwar et al. [9] demonstrated that patients with two or more catheters positioned per guidelines $(n=24)$ had an improved median survival of 55.6 weeks $(95 \%$ CI, 21.0 45.9) compared to 37.4 weeks (95\% CI, 21.0-45.9) for patients with fewer than two catheters optimally positioned $(n=19)$. In this analysis, we set out to determine if these results based on phase I studies can be replicated and to examine whether the additional imaging variables we collected may be predictive of clinical outcome. Table 8 summarizes the individual effect of the overall placement score, catheter positioning score, imaging grading score and local tumor control on clinical outcome (OS and PFS) based on the Cox PH model adjusting for age and KPS. In this larger, multicenter study we failed to demonstrate an effect of the catheter positioning score on OS $(P=0.53)$ or PFS $(P=0.72)$. Patients with two or more catheters optimally positioned $(n=84)$ had a median OS survival of 39.9 weeks (95\% CI, 32.6-52.4), compared to a median OS of 45.6 weeks (95\% CI, 36.4-65.0) among those with

Table 7 Correlative analysis of relationship among catheter positioning score, local tumor control and imaging change score on day 43 and day 71 MRI evaluation

\begin{tabular}{|c|c|c|c|c|c|c|c|c|c|}
\hline \multirow[t]{2}{*}{ Outcome } & \multirow[t]{2}{*}{ Predictor } & \multicolumn{4}{|c|}{ Day 43} & \multicolumn{4}{|c|}{ Day 71} \\
\hline & & \# Pts & \# Catheters & OR (95\% C.I.) & $P$-value & \# Pts & \# Catheters & OR (05\% C.I.) & $P$-value \\
\hline \multirow[t]{2}{*}{ Tumor control $^{\mathrm{a}}$} & Catheter score & 116 & 371 & & 0.61 & 101 & 321 & & 0.66 \\
\hline & 2 vs. $0 / 1$ & & & $0.79(0.32-1.94)$ & & & & $1.22(0.50-2.99)$ & \\
\hline \multirow[t]{4}{*}{ Tumor control $^{\mathrm{b}}$} & Imaging change score & 115 & 352 & & 0.20 & 98 & 287 & & 0.24 \\
\hline & 1 vs. 0 & & & $0.13(0.01-3.04)$ & & & & $0.09(0-10.4)$ & \\
\hline & 2 vs. 0 & & & $0.05(0-1.15)$ & & & & $0.02(0-2.66)$ & \\
\hline & $3 / 4$ vs. 0 & & & $0.09(0-2.68)$ & & & & $0.01(0-2.96)$ & \\
\hline \multirow[t]{2}{*}{ Imaging change score ${ }^{c}$} & Catheter score & 112 & 341 & & 0.86 & 99 & 289 & & 0.014 \\
\hline & 2 vs. $0 / 1$ & & & $0.97(0.73-1.29)$ & & & & $1.47(1.08-2.00)$ & \\
\hline
\end{tabular}

\footnotetext{
a,b OR represents the odds of achieving local tumor control comparing higher scores vs. lower scores of the corresponding predictors

${ }^{c}$ Imaging change scores 3 and 4 are combined due to limited number of catheters scored 4

OR (based on proportional odds model) represents the odds of achieving higher imaging change score comparing higher catheter positioning scores $(=2)$ vs. lower catheter positioning scores $(0$ or 1$)$

OR odds ratio, C.I. confidence interval
} 
Table 8 Cox proportional hazard model for PFS and OS

\begin{tabular}{|c|c|c|c|c|c|c|c|c|}
\hline & \multicolumn{4}{|l|}{ PFS } & \multicolumn{4}{|l|}{ OS } \\
\hline & \# pts & \# events & $\mathrm{HR}(95 \% \mathrm{CI})$ & $P$ & \# pts & \# events & $\mathrm{HR}(95 \% \mathrm{CI})$ & $P$ \\
\hline Overall catheter placement ${ }^{\mathrm{a}}$ & 153 & 140 & $0.85(0.61-1.20)$ & 0.35 & 153 & 119 & $0.89(0.62-1.29)$ & 0.55 \\
\hline Catheter Positioning score ${ }^{\mathrm{b}}$ & 154 & 141 & $1.06(0.76-1.48)$ & 0.72 & 154 & 119 & $1.13(0.78-1.63)$ & 0.53 \\
\hline \multicolumn{9}{|l|}{ Image change score ${ }^{c}$} \\
\hline Day $43^{\mathrm{e}}$ & 107 & 97 & $1.12(0.73-1.71)$ & 0.60 & 115 & 86 & $1.03(0.67-1.60)$ & 0.88 \\
\hline Day $71^{\mathrm{f}}$ & 75 & 66 & $1.53(0.88-2.66)$ & 0.13 & 96 & 72 & $1.39(0.84-2.28)$ & 0.20 \\
\hline \multicolumn{9}{|l|}{ Local tumor control $^{\mathrm{d}}$} \\
\hline Day $43^{\mathrm{e}}$ & 107 & 97 & $0.44(0.27-0.71)$ & $0.001 *$ & 119 & 92 & $0.56(0.35-0.88)$ & $0.01 *$ \\
\hline Day $71^{\mathrm{f}}$ & 78 & 68 & $0.81(0.43-1.52)$ & 0.51 & 100 & 75 & $0.81(0.48-1.38)$ & 0.44 \\
\hline
\end{tabular}

* Indicates significant $P$-value

${ }^{\text {a }}$ Fully confirming (all criteria fulfilled) vs. partially confirming/non-confirming

${ }^{\mathrm{b}}$ Patients with $\geq 2$ optimally placed catheters vs. patients with $\leq 1$ optimally placed catheters

${ }^{c}$ Patients with $\geq 2$ catheters graded with imaging change score 2 or above vs. patients with $\leq 1$ catheter graded with imaging change score 2 or above

${ }^{d}$ Patients with all catheters achieving local tumor control patients vs. patients with at least one catheter assessed as local tumor failure

e PFS analysis excluded patients who progressed by Day 43 and OS analysis excluded patients who died by Day 43

${ }^{\mathrm{f}}$ PFS analysis excluded patients who progressed by Day 71 and OS analysis excluded patients who died by Day 71

Note: All hazard ratios (HR) represent the hazard of the clinical endpoint occurring (i.e. progression or death) comparing higher scores vs. lower scores of the corresponding predictors. All $P$-values are associated with corresponding imaging variable after adjusting for age and KPS

fewer than two optimally positioned catheters $(n=70)$. Figure 1 depicts the Kaplan-Meier Curves for OS and PFS by catheter score. We further restricted the analysis to include only patients with ideal overall placement (fully conforming) and two or more optimal catheter positioning scores. Among these 45 patients, the median OS was 38.1 weeks (95\% CI, 30.9-49.4) and median PFS was 18.6 weeks (95\% CI, 15.6-25.3). The median OS is considerably less than that reported by Kunwar et al. among patients who had two or more optimally positioned catheters. In the analysis from Sampson et al. the definition of good catheter scores was slightly different including also scores of 1 . The authors found a significant effect on PFS after adjusting for KPS and age in patients with two or more catheters with a good positioning score. No effect was found on OS, which is in concordance with our findings. However, in this analysis we were unable to find a significant effect on PFS, which was not a designated endpoint in the PRECISE trial and therefore no central review was performed [17].

The imaging change score had no effect on OS or PFS in the Cox PH analysis after adjusting for age and KPS (OS: $P=0.88$ on day 43 and $P=0.20$ on day 71; PFS: $P=0.60$ on day $43 \mathrm{MRI}$ and $P=0.13$ on day $71 \mathrm{MRI}$ ). As expected, tumor control on day 43 was predictive of OS $(P=0.01)$ and PFS $(P=0.001)$. However, we were unable to detect a statistical significant correlation on day 71 MRI possibly due to the smaller in sample size. Figure 2
Fig. 1 OS and PFS for patients treated in the PRECISE study with $\mathrm{CB}$ by $\mathrm{CED}$ by catheter score
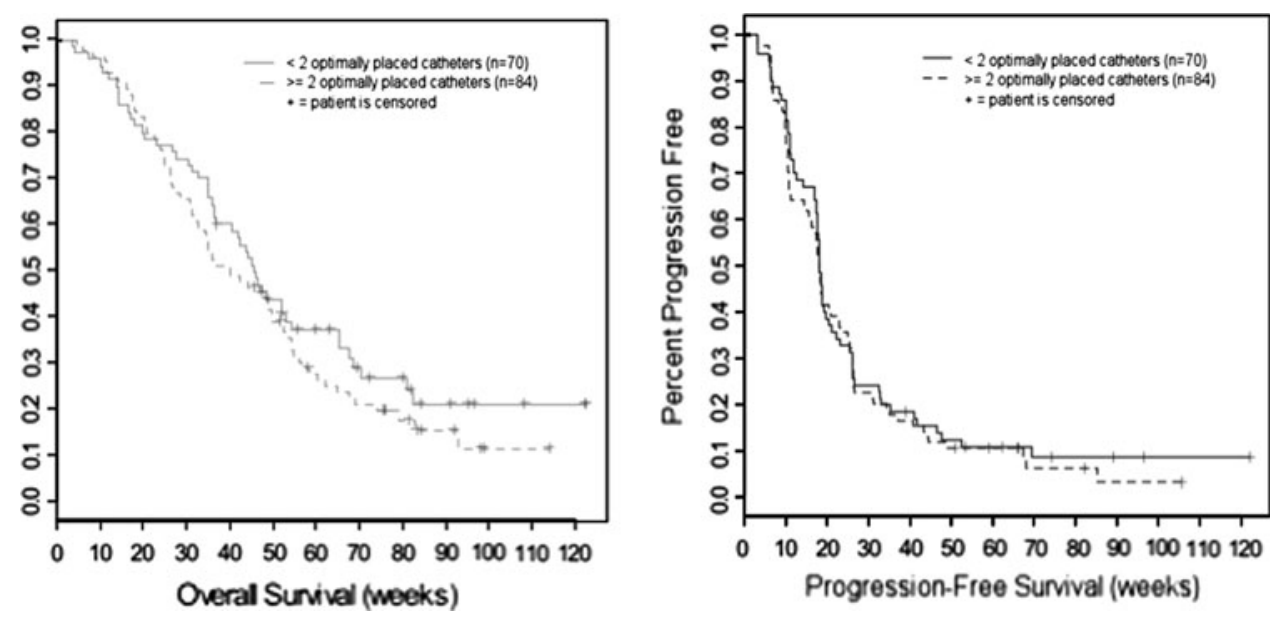
Fig. 2 Effect of local tumor on day 43 and day 71 MRI on OS and PFS. + Indicates censored observations. Note "Local failure" represents subjects with at least one local catheter failure. "No local failure" represents subjects that achieved local tumor control in all catheters
Day 43

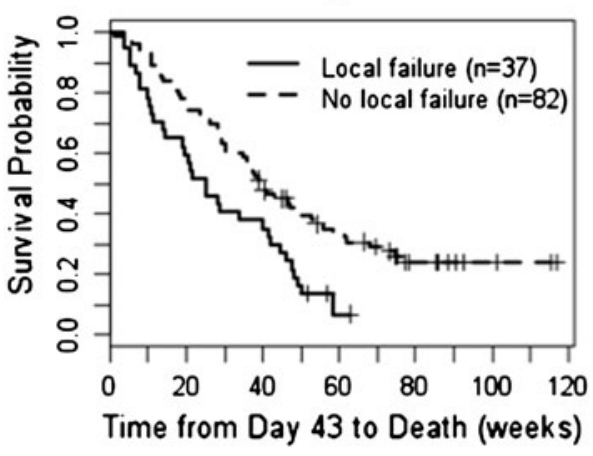

Day 71

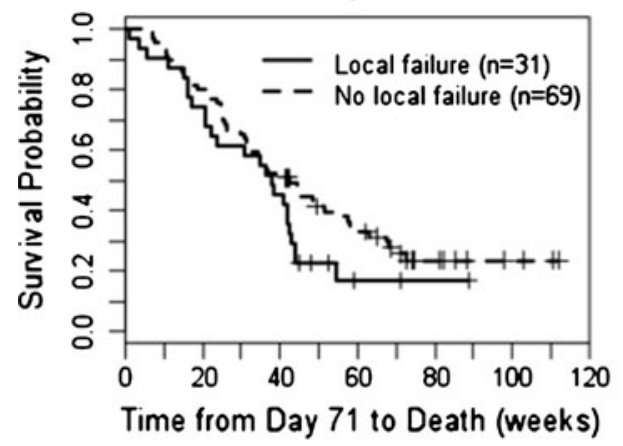

Day 43

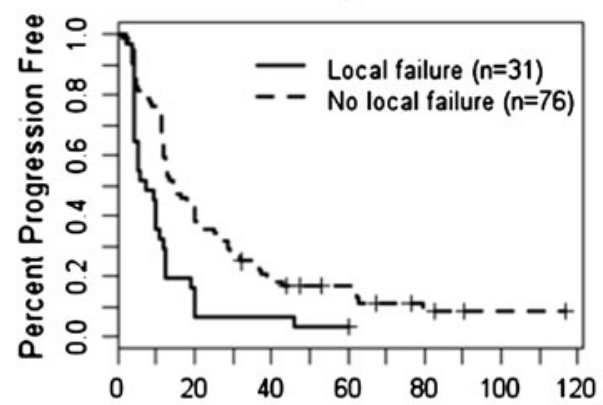

Time from Day 43 to Progression (weeks)

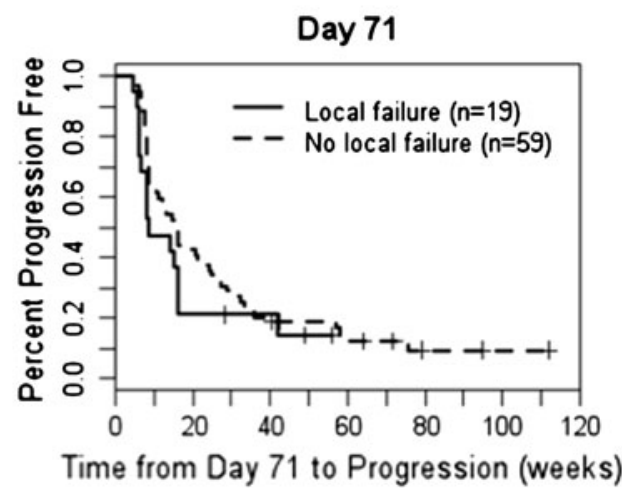

shows the Kaplan-Meier survival curves for OS and PFS by local tumor control status based on day 43 and day 71 MRI landmark analyses.

\section{Discussion}

The PRECISE trial is the largest study to date using CED for the delivery of a genetically engineered therapeutic agent for the treatment of patients with recurrent GBM. The study did not meet its primary endpoint by not showing a significant $O S$ difference between $C B$ via $C E D$ and GW. PFS was not a primary endpoint if this trial and not centrally reviewed [12]. To further examine the underlying reasons for this outcome, despite the very promising phase I study results, we investigated imaging characteristics on MRI scans obtained during this study. We assessed catheter positioning scores, overall catheter placement as well as imaging change scores for each MRI scan obtained during the study and correlated the collected variables with clinical outcome.

We found that $11 \%$ of patients did not fulfill the study inclusion criteria of GTR defined as greater than $95 \%$ resection of the contrast-enhancing mass and only a small percentage of patients (27\%) had a complete $(100 \%)$ resection of their enhancing tumor based on our review of the images. We excluded patients who did not fulfill the inclusion criteria of GTR. Further, the definition of extent of resection did not incorporate changes in T2/FLAIR signal abnormality, which potentially characterize significant metabolic and/or tumor burden. As such, the degree of variations in extent of resection may have influenced the study outcome negatively [18].

A detailed catheter scoring system was developed prior to study initiation based on studies using SPECT and ${ }^{123}$ I-HAS (Plasbumin). These studies showed that using CED could provide clinically relevant intracerebral distributions of high molecular weight compounds if catheters are accurately placed [14]. The overall placement score was created to determine placement in respect to resection cavity, T2/FLAIR signal abnormality and other catheters, whereas the catheter positioning score itself reflects the distance of each catheter in relation to different brain surfaces. The treating neurosurgeons underwent training for this complex treatment plan and also received feedback after catheter placement. Despite these specifications only $54 \%$ of the catheters were fully conformed regarding their overall placement and only $51 \%$ of all catheters were accurately positioned. These numbers indicate that the use of CED as a delivery technique is a highly specialized procedure and implementation of strict procedures is required to ensure accurate placement. An ad hoc analysis of the PRECISE trial showed centers that enrolled more than six patients had a higher median survival than centers that enrolled fewer patients. More experience with catheter placement by the treating neurosurgeon has been linked to improved survival 
[17]. This indicates that the training provided for CED in this study may have been insufficient and should be taken into consideration when planning future trials. It might also be beneficial to restrict this kind of complex delivery technique to centers of excellence to ensure the best possible outcome. Further to bear in mind is that patients treated on the PRECISE trial only received one infusion of the $\mathrm{CB}$. If repeated administration of the agent via CED would be surgically feasible the benefit on clinical outcome might be more substantial than given the drug only once.

In this analysis, we found that local tumor control status based on day 43 MRI was significantly predictive of OS and as expected of PFS. We were unable to establish this relationship based on day 71 data potentially due to the smaller sample size. Nevertheless, this finding is consistent with the general belief of clinicians that delay in tumor progression indicates the likelihood of prolonged survival. In fact, a recent study based on the experience of the North American Brain Tumor Consortium (NABTC) demonstrates a strong correlation between progression status at 6 months and OS among adult patients with recurrent high grade glioma [19].

Real time monitoring of drug distribution was not provided during the PRECISE study and we therefore used the catheter positioning score and imaging change score as surrogates. We were unable to find any correlation between catheter positioning score, local tumor control, and clinical outcome. In a subset of patients treated on the PRECISE trial, the distribution of the drug was estimated by using the iPlan Flow software (BrainLab AG, Germany) [17]. The authors found that there was a significant correlation between coverage volumes and overall catheter placement score. However, no correlation was found between coverage volumes and PFS or OS [17]. Additionally, these results are in contradiction to the analysis of prior phase I studies, which showed that OS was linked to adequate catheter placement. The median survival in the PRECISE study for patients treated with $\mathrm{CB}$ was reported to be 45.3 weeks, which is in concordance with prior studies [9]. Conversely, the median survival for patients with good catheter scores in the PRECISE trial was significantly lower (39.9 weeks with 95\% CI, 32.6-52.4) compared to prior phase I trials (55.6 weeks with 95\% CI, 21.0-45.9). In this analysis we used the same parameters to define good catheter scores as that used in the phase I analysis (two or more catheters with an optimal catheter positioning score of 2). We further investigated if the overall placement score affected these results. However, even patients with good overall placement score and good catheter scores had only a median survival of 38.1 weeks (95\% CI, 30.949.4 weeks). These results indicate that the catheter positioning score and overall placement score were not sufficient to predict response to therapy in the PRECISE trial.
Other techniques are needed to predict accurately the drug distribution in vivo. Recently, gadoteridol-loaded liposomes were shown to be successfully delivered in a canine model via CED and this technology also allows for monitoring of the distribution of these particles by real time imaging [20]. Using this technique, a recent study from Varenika et al. [21] showed that leakage occurred in $20 \%$ of infusions in monkeys. The clinical development of such a technique would identify patients in whom leakage occurred during the infusion or who showed poor drug distribution. Further trials using CED as a delivery method should incorporate detailed analysis of drug distribution to determine if delivery of the drug is adequate and covers the expected area. Monitoring drug distribution will also help to further identify additional factors needed to provide adequate drug delivery, which is not currently captured in the catheter positioning score or overall placement score.

Besides drug distribution, drug specificity and expression of the target molecule on tumor cells influence the response to therapy. With respect to target expression, several studies have shown that the target of $\mathrm{CB}$, the IL-13R, is over-expressed in a large number of high grade glioma explants $[6,22]$. A recent study using a commercially available tissue array and IL-13R antibody showed that up to $75 \%$ of patients with GBM over-express the IL-13R. This will allow upfront testing for future trials and should be included in the eligibility criteria [23]. The percentage of IL13R positive samples within the PRECISE cohort is currently not known and might have influenced the outcome. Heterogeneous expression of the IL-13R has been demonstrated in tumor samples as well as variation in the expression from various sites of tumor, however the use of different antibodies used in these studies makes it difficult to assess the real impact of these findings [6, 22-25]. Given that most patients will undergo surgery at recurrence, the expression of the target gene at the tumor margins is much more crucial than the expression in the core of the tumor. Further analysis of the expression of the IL-13R in samples obtained during this trial will help to answer some of these questions. In summary, more rigorous upfront biological based inclusion criteria might be needed when using a targeted therapy like CB.

$\mathrm{CB}$ related imaging changes seen during the PRECISE trial were similar to what has been reported from phase I studies. We used the imaging change score as a marker for drug distribution based on a study that showed that new FLAIR/T2 signal changes are correlated with drug distribution [11]. We were unable to identify any correlation between the imaging change score, local tumor control or clinical outcome. The numbers of catheters associated with more extensive imaging changes were relatively low and therefore we might have lacked the statistical power to see any differences. 


\section{Conclusion}

The PRECISE study did not demonstrate a survival benefit of patients treated with $\mathrm{CB}$ versus $\mathrm{GW}$ in resectable recurrent GBM. Despite intensive on site training a majority of catheters were not positioned correctly highlighting the complexity of this technique. Detailed analysis of catheter positioning score, overall placement score and imaging change score did not reveal any correlation of these parameters with clinical outcome and better methods for determining drug distribution by CED need to be included in future study designs. Further analysis of the target expression will be important to further identify areas of improvement.

Acknowledgements This work was supported by the PO grant PO1CA118816-02, Campini Foundation (SM) as well by BrainLab AG, Germany. The PRECISE trial was sponsored by NeoPharm, Inc.

Open Access This article is distributed under the terms of the Creative Commons Attribution Noncommercial License which permits any noncommercial use, distribution, and reproduction in any medium, provided the original author(s) and source are credited.

\section{Appendix}

The following institutions and investigators participated in the trial: City of Hope Medical Center, Duarte, California (Behnam Badie); Ohio State University Medical Center, Columbus, Ohio (Antonio Chiocca); H. Lee Moffitt Cancer Center, Tampa, Florida (Frank Vrinis); University of Hamburg-Eppendorf, Hamburg, Germany (M.W.); University of Schleswig-Holstein, Lubeck, Germany (Maximillian H. Mehdorn); Sheba Medical Center, Tel Hashomer, Israel (Zvi Cohen); Tel Aviv Sourasky Medical Center, Tel Aviv, Israel (Z.R.); The Cleveland Clinic Foundation, Cleveland, Ohio (G.B.); University of Colorado Hospital-Medical School, Aurora, Colorado (Kevin Lillehei); Hospital of the University of Pennsylvania, Philadelphia, Pennsylvania (Kevin Judy); Baylor College of Medicine, Houston, Texas (Pamela New); Emory University, Atlanta, Georgia (Jeffrey Olson); Henry Ford Health Systems, Detroit, Michigan (Tom Mikkelsen); University of Alabama at Birmingham, Birmingham, Alabama (James Markert); Wake Forest University Health Sciences, Winston-Salem, North Carolina (Stephen Tatter); John Hopkins Hospital, Baltimore, Maryland (Jon Weingart); The University of Texas M.D. Anderson Cancer Center, Houston, Texas (Fredrick Lang); Yale University School of Medicine, New Haven, Connecticut (J.P.); University of California-San Francisco, San Francisco, California (M.P.); Memorial Sloan Kettering Cancer Center, New York, New York (Lauren Abrey); Duke
University Medical Center, Durham, North Carolina (David Reardon); Carolina Neurosurgery and Spine Associates, Charlotte, North Carolina (Anthony Asher); Dana Farber Cancer Institute, Boston, Massachusetts (Peter Black); Northwestern Medical Faculty Foundations, Inc., Chicago, Illinois (James Chandler); Evanston Northwestern Healthcare, Evanston, IL, USA (Nina Paleologos); University of Wisconsin Hospital and Clinics, Madison, Wisconsin (John Kuo); Cedar Sinai Medical Center, Neurosurgical Institute, Los Angeles, California (John Yu); CINN at Rush University Medical Center, Chicago, Illinois (Richard Byrne); Medical University of South Carolina, Charleston, South Carolina (Sunil Patel); University of Virginia Health Systems, Charlottesville, Virginia (M.S.); Florida Hospital Neuroscience Institute, Orlando, Florida (Edward Pan); University of California-Los Angeles, Los Angeles, California (Timothy Cloughesy); Weill Cornell Medical College, Department of Neurological Surgery, New York, New York (Theodore Schwartz); University of Chicago Medical Center, Chicago, Illinois (Macieij Lesniak); Columbia University Medical Center, New York, New York (Jeffrey Bruce); University of Southern California, Los Angeles, California (Thomas Chen); Montreal Neurological Institute and Hospital, Montreal, Quebec, Canada (Rolando Del Maestro); Cancer Care Manitoba, Winnipeg, Manitoba, Canada (David Eisenstat); Royal University Hospital, Saskatoon, Saskatchewan, Canada (Daryl Fourney); Toronto Western Hospital, Toronto, Ontario, Canada (Abhijit Guha); University of Utah, Salt Lake City, Utah (Randy L. Jensen); London Regional Cancer Program, London, Ontario, Canada (David MacDonald); Walter Mackenzie Health Sciences Center, Edmonton, Alberta, Canada (Vivek Mehta); University Hospital Groningen, Groningen, The Netherlands (Jan Jakob A. Mooij); Sunnybrook and Women's College Health Sciences Centre, Toronto, Ontario, Canada (Farhad Pirouzmand); Institute of Neurological Sciences, Southern General Hospital, Glasgow, UK (Laurence T. Dunn); Technische Universitat Dresden Klinic und Poliklinik fur Neuochirugie, Dresden, Germany (Gabriele Schackert); Ludwig-Maximilians Universitat Munchen, Munich, Germany (Roland Goldbrunner); Mayo Clinic, Rochester, Minnesota (Joon Uhm); University of Heidelberg, Heidelberg, Germany (Andreas Unterberg); Erasmus University Medical Center, Rotterdam, The Netherlands (John G. Wolbers); Rabin Medical Center, Petah Tikva, Israel (Zvi Harry Rappaport); Calgary Health Region, Calgary, Alberta, Canada (Mark Hamilton); Baptist Memorial Hospital, Memphis, Tennessee (Bruce Frankel); West Virginia University, Robert C. Byrd Health Sciences Center, Mograntown, West Virginia (Warren Boling); St Louis University, St Louis, Missouri (Richard Bucholz); University of Texas Southwestern Medical Center, Dallas, 
Texas (Bruce Mickey); Oregon Health and Science University, Portland, Oregon (Johhny Delashaw Jr); Virginia Mason Clinic, Seattle, Washington (Lynne Taylor).

\section{References}

1. Stupp R, Dietrich PY, Ostermann Kraljevic S, Pica A, Maillard I, Maeder P, Meuli R, Janzer R, Pizzolato G, Miralbell R, Porchet F, Regli L, de Tribolet N, Mirimanoff RO, Leyvraz S (2002) Promising survival for patients with newly diagnosed glioblastoma multiforme treated with concomitant radiation plus temozolomide followed by adjuvant temozolomide. J Clin Oncol 20:1375-1382

2. Sampson JH, Akabani G, Archer GE, Berger MS, Coleman RE, Friedman AH, Friedman HS, Greer K, Herndon JE 2nd, Kunwar S, McLendon RE, Paolino A, Petry NA, Provenzale JM, Reardon DA, Wong TZ, Zalutsky MR, Pastan I, Bigner DD (2008) Intracerebral infusion of an EGFR-targeted toxin in recurrent malignant brain tumors. Neuro Oncol 10:320-329

3. Vogelbaum MA, Sampson JH, Kunwar S, Chang SM, Shaffrey M, Asher AL, Lang FF, Croteau D, Parker K, Grahn AY, Sherman JW, Husain SR, Puri RK (2008) Convection-enhanced delivery of cintredekin besudotox (interleukin-13-Pe38qqr) followed by radiation therapy with and without temozolomide in newly diagnosed malignant gliomas: phase 1 study of final safety results. Neurosurgery 61(5):1031-1037

4. Debinski W, Obiri NI, Pastan I, Puri RK (1995) A novel chimeric protein composed of interleukin 13 and Pseudomonas exotoxin is highly cytotoxic to human carcinoma cells expressing receptors for interleukin 13 and interleukin 4. J Biol Chem 270:16775-16780

5. Husain SR, Joshi BH, Puri RK (2001) Interleukin-13 receptor as a unique target for anti-glioblastoma therapy. Int $\mathbf{J}$ Cancer 92: 168-175

6. Joshi BH, Plautz GE, Puri RK (2000) Interleukin-13 receptor alpha chain: a novel tumor-associated transmembrane protein in primary explants of human malignant gliomas. Cancer Res 60: $1168-1172$

7. Liu H, Jacobs BS, Liu J, Prayson RA, Estes ML, Barnett GH, Barna BP (2000) Interleukin-13 sensitivity and receptor phenotypes of human glial cell lines: non-neoplastic glia and low-grade astrocytoma differ from malignant glioma. Cancer Immunol Immunother 49:319-324

8. Sampson JH, Raghavan R, Brady ML, Provenzale JM, Herndon JE 2nd, Croteau D, Friedman AH, Reardon DA, Coleman RE, Wong T, Bigner DD, Pastan I, Rodriguez-Ponce MI, Tanner P, Puri R, Pedain C (2007) Clinical utility of a patient-specific algorithm for simulating intracerebral drug infusions. Neuro Oncol 9:343-353

9. Kunwar S, Prados MD, Chang SM, Berger MS, Lang FF, Piepmeier JM, Sampson JH, Ram Z, Gutin PH, Gibbons RD, Aldape KD, Croteau DJ, Sherman JW, Puri RK (2007) Direct intracerebral delivery of cintredekin besudotox (IL13-PE38QQR) in recurrent malignant glioma: a report by the Cintredekin Besudotox Intraparenchymal Study Group. J Clin Oncol 25:837-844

10. Parney IF, Kunwar S, McDermott M, Berger M, Prados M, Cha S, Croteau D, Puri RK, Chang SM (2005) Neuroradiographic changes following convection-enhanced delivery of the recombinant cytotoxin interleukin $13-\mathrm{PE} 38 \mathrm{QQR}$ for recurrent malignant glioma. J Neurosurg 102:267-275

11. Sampson JH, Raghavan R, Provenzale JM, Croteau D, Reardon DA, Coleman RE, Rodriguez Ponce I, Pastan I, Puri RK, Pedain C (2007) Induction of hyperintense signal on T2-weighted MR images correlates with infusion distribution from intracerebral convection-enhanced delivery of a tumor-targeted cytotoxin. AJR Am J Roentgenol 188:703-709

12. Kunwar S, Chang S, Westphal M, Vogelbaum M, Sampson J, Barnett G, Shaffrey M, Ram Z, Piepmeier J, Prados M, Croteau D, Pedain C, Leland P, Husain SR, Joshi BH, Puri RK, PRECISE Study Group (2010) Phase III randomized trial of CED of IL13PE38QQR vs Gliadel wafers for recurrent glioblastoma. Neuro Oncol. doi:10.1093/neuonc/nop054

13. Raghavan R, Brady ML, Rodriguez-Ponce MI, Hartlep A, Pedain C, Sampson JH (2006) Convection-enhanced delivery of therapeutics for brain disease, and its optimization. Neurosurg Focus 20:E12

14. Sampson JH, Brady ML, Petry NA, Croteau D, Friedman AH, Friedman HS, Wong T, Bigner DD, Pastan I, Puri RK, Pedain C (2007) Intracerebral infusate distribution by convection-enhanced delivery in humans with malignant gliomas: descriptive effects of target anatomy and catheter positioning. Neurosurgery 60: ONS89-98 (discussion ONS98-89)

15. Rabe-Hesketh S, Skrondal A (2005) Multilevel and longitudinal modeling using stata, 2nd edn. Stata Press, College Station

16. Therneau TM, Grambsch PM (2001) Modeling survival data: extending the Cox model, 1st edn. Springer, New York

17. Sampson JH, Archer G, Pedain C, Wembacher-Schroder E, Westphal M, Kunwar S, Vogelbaum MA, Coan A, Herndon JE, Raghavan R, Brady ML, Reardon DA, Friedman AH, Friedman HS, Rodriguez-Ponce MI, Chang SM, Mittermeyer S, Croteau D, Puri RK (2009) Poor drug distribution as a possible explanation for the results of the PRECISE trial. J Neurosurg

18. Lacroix M, Abi-Said D, Fourney DR, Gokaslan ZL, Shi W, DeMonte F, Lang FF, McCutcheon IE, Hassenbusch SJ, Holland E, Hess K, Michael C, Miller D, Sawaya R (2001) A multivariate analysis of 416 patients with glioblastoma multiforme: prognosis, extent of resection, and survival. J Neurosurg 95:190-198

19. Lamborn KR, Yung WK, Chang SM, Wen PY, Cloughesy TF, DeAngelis LM, Robins HI, Lieberman FS, Fine HA, Fink KL, Junck L, Abrey L, Gilbert MR, Mehta M, Kuhn JG, Aldape KD, Hibberts J, Peterson PM, Prados MD (2008) Progression-free survival: an important end point in evaluating therapy for recurrent high-grade gliomas. Neuro Oncol 10:162-170

20. Dickinson PJ, LeCouteur RA, Higgins RJ, Bringas JR, Roberts B, Larson RF, Yamashita Y, Krauze M, Noble CO, Drummond D, Kirpotin DB, Park JW, Berger MS, Bankiewicz KS (2008) Canine model of convection-enhanced delivery of liposomes containing CPT-11 monitored with real-time magnetic resonance imaging: laboratory investigation. J Neurosurg 108:989-998

21. Varenika V, Dickinson P, Bringas J, LeCouteur R, Higgins R, Park J, Fiandaca M, Berger M, Sampson J, Bankiewicz K (2008) Detection of infusate leakage in the brain using real-time imaging of convection-enhanced delivery. J Neurosurg 109:874-880

22. Debinski W, Gibo DM, Slagle B, Powers SK, Gillespie GY (1999) Receptor for interleukin 13 is abundantly and specifically over-expressed in patients with glioblastoma multiforme. Int $\mathrm{J}$ Oncol 15:481-486

23. Wykosky J, Gibo DM, Stanton C, Debinski W (2008) Interleukin13 receptor alpha 2, EphA2, and Fos-related antigen 1 as molecular denominators of high-grade astrocytomas and specific targets for combinatorial therapy. Clin Cancer Res 14(1):199-208

24. Jarboe JS, Johnson KR, Choi Y, Lonser RR, Park JK (2007) Expression of interleukin-13 receptor alpha2 in glioblastoma multiforme: implications for targeted therapies. Cancer Res 67:7983-7986

25. Saikali S, Avril T, Collet B, Hamlat A, Bansard JY, Drenou B, Guegan Y, Quillien V (2007) Expression of nine tumour antigens in a series of human glioblastoma multiforme: interest of EGFRvIII, IL-13Ralpha2, gp100 and TRP-2 for immunotherapy. J Neurooncol 81:139-148 